\title{
TAUBERIAN CONDITIONS FOR CONULL SPACES
}

\section{J. CONNOR}

\author{
Department of Mathematical Sciences \\ Kent State University \\ Kent, Ohio 44242 U.S.A. \\ and
}

\section{A. K. SNYDER}

Department of Mathematics

Lehigh University

Bethlehem, PA 18015 U.S.A.

(Received February 15, 1984)

ABSTRACT. The typical Tauberian theorem asserts that a particular summability method cannot map any divergent member of a given set of sequences into a convergent sequence. These sets of sequences are typically defined by an "order growth" or "gap" condition. We establish that any conull space contains a bounded divergent member of such a set; hence, such sets fail to generate Tauberian theorems for conull spaces.

KEY WORDS AND PHRASES. FK UNACE; conuZZ.

1380 MATLEMATICS SUBJECT CLASSIFICATION CODE. 46A45, 40E05, 40HO5.

\section{INTRODUCTION}

In this note we establish that a broad class of Tauberian conditions that hold for regular matrix methods cannot hold for conull methods. In particular, we consider "gap" and "order growth" conditions and show they are not Tauberian conditions for conul1 spaces.

Before proceeding with the discussion, we pause to collect some definitions and theorems. We let

$$
\begin{aligned}
& \omega=\{\text { the set of all sequences }\} \\
& \phi=\{x \in \omega: \quad x \text { is finitely nonzero }\} \\
& c_{0}=\left\{x \in \omega: \quad \text { imit } x_{n}=0\right\} \\
& c=\left\{x \in \omega: \quad \text { imit } x_{n} \text { exists }\right\} \\
& e^{\infty}=\left\{x \in \omega: \sup _{n}\left|x_{n}\right|<\infty\right\} \\
& c=(1,1,1, \ldots) \\
& e^{n}=\text { the nth unit vector, } \sigma^{n}=\sum_{j=1}^{n} e^{j}
\end{aligned}
$$

and, if $E$ is a Frechet space, $E^{\prime}$ denotes its continuous dual.

Recall that a locally convex Frechet space is FK-space if it is a vector subspace of $\omega$ and the coordinate functionals are conti nuous. We call an FK-space a sequence space if it contains $\phi$. 
Definition: A sequence space $E$ is (a) conservative if $E=c$, (b) semi-conservative if $\left(\sigma^{n}\right)$ is weakly Cauchy, and (c) conull if $\left(\sigma^{n}\right)$ converges weakly to e.

Clealy conull spaces are semi-conservative. It is known that a sequence space

E contains $c_{0}$ if and only if $\sum_{j=1}^{\infty}\left|f\left(e^{j}\right)\right|<\infty$ for all $f \in E^{\prime}[1]$, hence conservative spaces are also semi-conservative.

Definition: An FK-space is pre-conull if every semi-conservative space containing it is conull.

We list some well-known facts in the following two theorems. ([2], [3]) Theorem: Let $\mathrm{E}, \mathrm{F}$ be FK-spaces with $\mathrm{E} \subseteq \mathrm{F}$ (set theoretically) and $A$ a matrix map. Then (a) the inclusion map from $E$ into $F$ is continuous; (b) $E_{A}=\{x \varepsilon \omega$ : $A x \in E\}$ is an FK-space; (c) if $E$ is conull, $F$ is conull; and (d) if $\phi \subset E$, $E$ is closed in $F$ and $F$ is conull, then $E$ is conull.

Theorem: The intersection of two conull spaces is conull.

We also use a characterization of conservative conull spaces. Let $r=\left(r_{n}\right)$ be be an increasing sequence of natural numbers with $r_{1}=1$. Define

$$
O_{n}^{r}(x)=\max \left\{\left|x_{u}-x_{v}\right|: r_{n} \leq u<v \leq r_{n+1}\right\}
$$

and set

$$
\Omega(r)=\left\{x \in \omega: \text { limit }{ }_{n} O_{n}^{r}(x)=0\right\} \text {. }
$$

If we define ||$x||_{r}=\left|x_{1}\right|+\sup _{n} o_{n}^{r}(x)$ for $x \varepsilon \Omega(r)$, then $\left(\Omega(r), \| \cdot||_{r}\right)$ is a conservative conull space. In fact, we also have from [3]:

Theorem: Let $\mathrm{E}$ be conservative. $\mathrm{E}$ is conull if and only if $\mathrm{E} \Omega \Omega(\mathrm{r})$ for some $\mathrm{r}$. Now we are ready to begin. Let $E$ be an FK-space and $P \subset \ell^{\infty}$. We say that "P is a Tauberian condition for $E^{\prime \prime}$ provided

(*) $x \in P \cap E$ implies $x \in c$.

Note that $(*)$ is the general form of a (matrix summability) Tauberian theorem. The candidates for playing the part of $P$ are defined as follows.

Definition: ("gap" conditions) Let $s=\left(s_{n}\right)$ be an increasing sequence of natural numbers; set

$$
G(s)=\left\{x \in \omega: \quad(\Delta x)_{k}=x_{k}-x_{k+1} \neq 0 \text { only if } k=s_{n} \text { for some } n \varepsilon N\right\} .
$$

Definition: ("order growth" conditions) Let $\lambda=\left(\lambda_{n}\right)$ be a sequence of positive real numbers such that $\stackrel{\sum}{n=1}_{n}^{\infty} \lambda_{n}=\infty$.

and

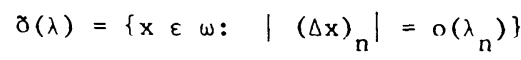

$$
\begin{aligned}
& \tilde{n}(\lambda)=\left\{x \in \omega:\left|(\Delta x)_{n}\right|=0\left(\lambda_{n}\right)\right\} \text {. }
\end{aligned}
$$

Recall that if $E$ is the FK-space of Caesaro summable sequences and we let $s=\left(s_{n}\right)$ satisfy limit $s_{n+l} / s_{n}>1$ and $\lambda_{n}=n^{-1}$, then $O(\lambda) \cap \ell^{\infty}$ and $G(s) n \ell^{\infty}$ are Tauberian conditions for E. Also, J. Fridy has shown that for any real regular matrix $\Lambda$ there is always an $s$ such that $G(s) \cap l^{\infty}$ is a Tauberian condition for the summability field of $A[4]$. 


\section{PRE-CONULL SPACES.}

Our arguments hinge on two properties of pre-conull spaces: that they are necessarily "large" and that pre-conullity is preserved under intersection with a conull space. These properties are exposed in the next two lemmas.

Lemma 1: If $E$ is pre-conull and $F$ is conull, then $E \cap F$ is pre-conull. Proof: First observe that if $\mathrm{H}$ is a vector space containing $\mathrm{E} \cap \mathrm{F}$, then $(\mathrm{E}+\mathrm{H} \cap \mathrm{F})$ $n F \subseteq H$. This follows from noting that if $y \varepsilon E, z \varepsilon H \cap F$ and $y+z \varepsilon F$, then $y \in E \cap F$, and clearly $E \cap F+H \cap F \subset H$.

Now suppose $H$ is a semi-conservative space containing $E \cap F$. Since $F$ is conull, F n $\mathrm{H}$ is semi-conservative (i.e., ( ${ }^{\mathrm{n}}$ ) is weakly Cauchy in $\mathrm{F} \cap \mathrm{H}$ ), and consequently $\mathrm{E}+\mathrm{F} \cap \mathrm{H}$ is semi-conservative. Now $\mathrm{E} \subseteq \mathrm{E}+\mathrm{F} \cap \mathrm{H}$ implies that $\mathrm{E}+\mathrm{F} \cap \mathrm{H}$ is conul1, and since $\mathrm{F}$ is conull, $(\mathrm{E}+\mathrm{F} \cap \mathrm{H}) \cap \mathrm{F}$ is conull. Consequently $\mathrm{H}$ is also conull and we have established the lemma.

Lemma 2: If $\mathrm{E}$ is pre-conull, then $\left(\mathrm{E} \cap \ell^{\infty}\right)+\mathrm{c} z \Omega(\mathrm{r}) \cap \ell^{\infty}$ for some $\mathrm{r}$. In particular, E contains a bounded divergent sequence.

Proof: It is easy to check that $\left(E \cap \ell^{\infty}\right)+c=(E+c) \cap \ell^{\infty}$.

Since $\mathrm{E}+\mathrm{C}$ is conservative, hence semi-conservative, and $\mathrm{E}$ is pre-conul1, $\mathrm{E}+\mathrm{C}$ is a conservative conull space. Consequently $\mathrm{E}+\mathrm{c}$ contains an $\Omega(\mathrm{r})$ for some $\mathrm{r}$ and $\left(E \cap \ell^{\infty}\right)+c-\Omega(r) \cap \ell^{\infty}$.

Now E contains a bounded divergent sequence since $\Omega(r)$ does, i.e., $\Omega(r)$ contains a bounded divergent sequence of the form $y+z$ where $y \varepsilon E \cap \ell^{\infty}$ and $z \varepsilon c$ and $y$ must be divergent (otherwise, $y+z \varepsilon c$ ).

We also give a sufficient condition for a space to be pre-conull. Lemma 3: E is pre-conull if there is a sequence $\left(z^{n}\right) \subset \alpha$ such that $z^{n}$ converges to e in $E$ and $\sup _{n} \overbrace{k=1}^{\infty}\left|\left(\Delta z^{n}\right)_{k}\right|<\infty$.

Proof: This follows from the fact that semi-conservative space $F$ is conull if (and only if) there is a sequence $\left(z^{n}\right) \subseteq \oint$ such that $z^{n}$ converges to $e$ in $F$ and $\sup _{n} \sum_{k=1}^{\infty}$ $\left|\left(\Delta z^{n}\right)_{k}\right|<\infty\lceil 5]$.

3. THE MAIN RESUlT.

Lemma 4: If $(i(s), \tilde{O}(\lambda)$ and $\delta(\lambda)$ are defined as in section 1 , they are all pre-conul1 spaces.

Proof: First we establish that $G(s)$ is a pre-conull space. Observe that $G(s)$ is a closed subspace of $\omega$ when $\omega$ is given the topology of coordinatewise convergence ( $\omega^{\prime} s$ FK-topology), hence it is an FK-space.

Now let $\mathrm{E}$ be any semi-conservative space containing $\mathrm{G}(\mathrm{s})$ and set

$$
\sigma^{n_{k}}=\sum_{j=1}^{s_{k}} e^{j}, k=1,2, \ldots .
$$

Since $\left(\sigma^{{ }^{n}}\right)$ converges to e in $G(s)$, it converges to e in $E$. Now we have that $\left(\sigma^{n}\right)$ is weakly Cauchy in $\mathrm{E}$ and has a subsequence which converges weakly to e, hence $\left(\sigma^{\mathrm{n}}\right)$ is weakly convergent to $\mathrm{e}$ in $\mathrm{E}$. Thus $\mathrm{E}$ is conull, and consequently, $\mathrm{G}(\mathrm{s})$ is pre-conull. 
We now turn our attention to $\delta(\lambda)$ and $\tilde{O}(\lambda)$. Consider the natrix $B=\left(b_{n, k}\right)$ defined by $b_{1,1}=1, b_{n, n-1}=\lambda_{n-1}^{-1}, b_{n, n}=-\lambda_{n-1}^{-1}$ and $b_{n, k}=0$ otherwise. Observe that $\tilde{O}(\lambda)=\ell_{B}^{\infty}$ and $\delta(\lambda)=\left(c_{0}\right)_{B}$, hence both are FK-spaces with the metric topology being given by the norm ||$x \|_{B}=\max \left\{\left|x_{1}\right|, \sup _{k} \lambda_{k}^{-1}\left|(\Delta x)_{k}\right|\right\}$ in both cases. Also note that, since $\delta(\lambda) \subseteq \tilde{O}(\lambda)$, it suffices to show that $\delta(\lambda)$ is pre-conull to show that $\tilde{O}(\lambda)$ is pre-conull.

We now construct a sequence in $x(\lambda)$ that satisfies the hypothesis of Lemma 3 . Let $\left(\beta_{k}\right)$ be a sequence of reals such that $0<\beta_{k}<1$ for all $k \varepsilon N, \sum_{k=1}^{\infty} \beta_{k}=\infty$ and $\operatorname{limit}_{k} \lambda_{k}^{-1} \beta_{k}=0$ (this is possible by one of Abel's results $[6, p .125]$ ), and set $I(n)=\max \left\{k: \sum_{i=n}^{k-1} \beta_{i}<1\right\}$. Note that $n<I(n)<\infty$ for all $n \varepsilon N$. Define $z^{n}$ by $z_{k}^{n}=$ for $k \leq n, z_{k}^{n}=1-\sum_{i=n}^{k-1} \beta_{i}$ for $n<k \leq I(n)$ and $z_{k}^{n}=0$ for $k>I(n)$. It's easy to check that ||$z^{n}-e \|_{B} \leq \max \left\{\left|\lambda_{k}^{-1} \beta_{k}\right|: n \leq k \leq I(n)\right\}$, hence 1imit $z^{n}=e$ in $\delta(\lambda)$. It's also clear that $\left(z^{n}\right) \subseteq \phi$ and $\sum_{k=1}^{\infty}\left|\left(\Delta z^{n}\right)_{k}\right|=1$ for all $n \in N$, hence, by Lemma 3 , $\sigma(\lambda)$ is pre-conull.

We can now show that neither $\delta(\lambda), \tilde{O}(\lambda)$ nor $G(s)$ can generate Tauberian conditions for a conull space.

Theorem: If $E$ is a conull space, then $\sigma(\lambda) \cap E, \tilde{O}(\lambda) \cap E$ and $G(s) \cap E$ all contain a bounded divergent sequence.

Proof: Since $\tilde{O}(\lambda), \tilde{o}(\lambda)$ and $G(s)$ are all pre-conull, $\tilde{O}(\lambda) \cap E, \sigma(\lambda) \cap E$ and $G(s) \cap E$ are all pre-conul1, hence each contain a bounded divergent sequence.

\section{REFERENCES}

1. BENNETT, G. and KALTON, N. J. "FK spaces containing c," Duke Math Jour., 39 (3) (1972), 561-582.

2. WILANSKY, A. "Summability through functional analysis," North-Holland, 1984.

3. SNYDER, A. K. "Conull and coregular FK spaces," Math. Zeitschr., 90 (1965), 378-381.

4. FRIDY, J. "Tauberian theorems via block dominated matrices," Pacific Journal of Math. 81 (1979), 81-91.

5. SNYDER, A. K. and WILANSKY, A. "Inclusion theorems and semi-conservative FK spaces," Rocky Mountain J. of Math. 2(4) (1972). 595-603.

6. KNOPP, K. Infinite Sequences and Series, Dover, 1956. 


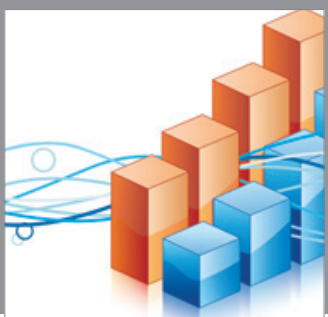

Advances in

Operations Research

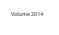

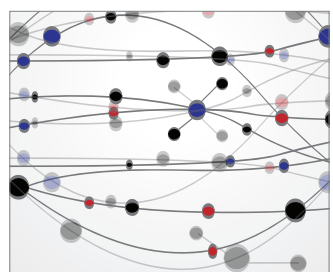

\section{The Scientific} World Journal
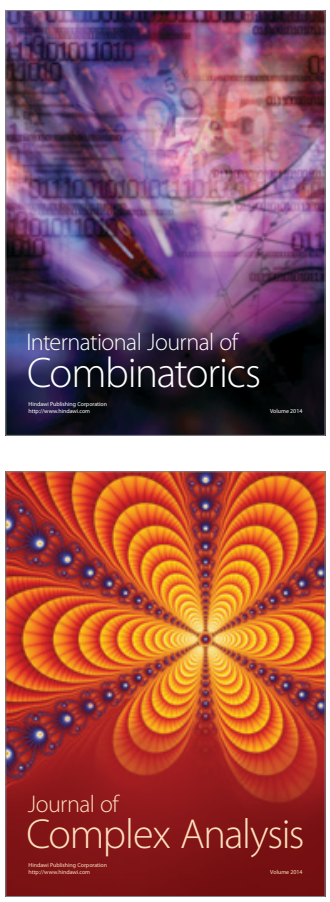

International Journal of

Mathematics and

Mathematical

Sciences
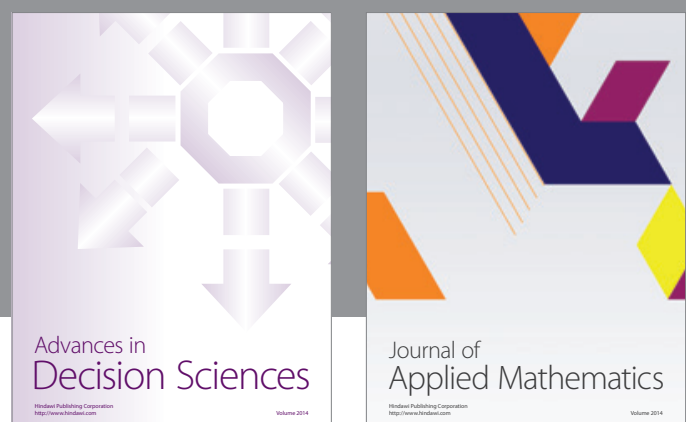

Journal of

Applied Mathematics
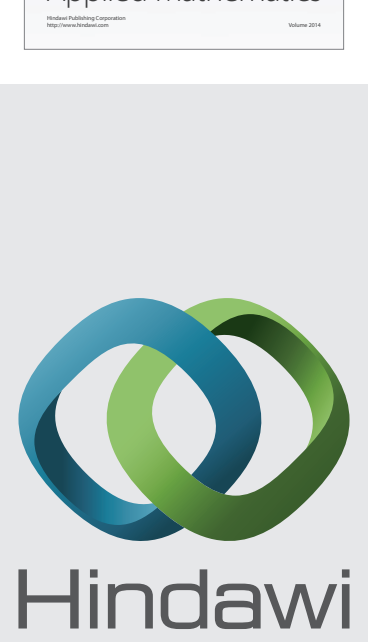

Submit your manuscripts at http://www.hindawi.com
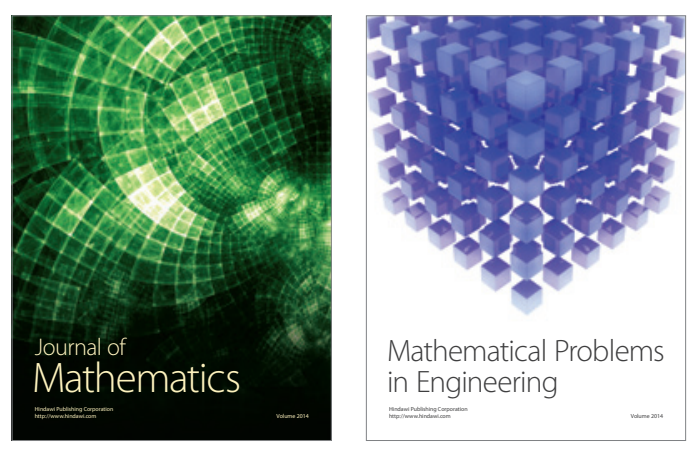

Mathematical Problems in Engineering


Journal of

Function Spaces
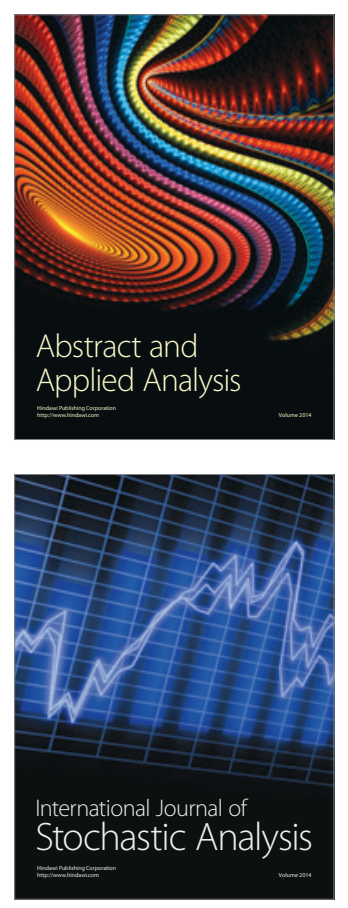

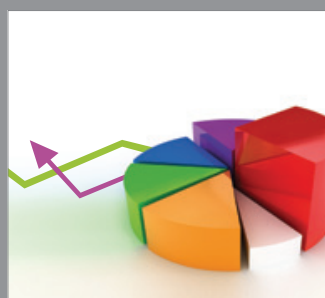

ournal of

Probability and Statistics

Promensencen
